\title{
Genetic Algorithm Combined with Ray Tracer for Optimizing Cell-Free mMIMO Topology in a Confined Environment
}

\author{
Ke Shen*, Toon De Pessemier*, Luc Martens*, Wout Joseph*, Yang Miao ${ }^{\dagger}$ \\ *Department of Information Technology, Ghent University/IMEC, Ghent, Belgium, ke.shen@ugent.be \\ ${ }^{\dagger}$ Faculty of Electrical Engineering, University of Twente, Enschede, the Netherlands, y.miao@utwente.nl
}

\begin{abstract}
This paper proposes a customized genetic algorithm (GA) to generate the optimal cell-free topology for multi-user massive MIMO (mMIMO) in a confined environment. As far as we know, it is beyond the literature and is the first attempt to apply GA in optimizing the base station (BS) antenna placement for cell-free mMIMO. The BS antennas' placement is encoded with an adjusted binary matrix representation, which is straightforward for the subsequent genetic operations. The explored candidates by GA can evolve beyond the parents, where the fitness of individuals is evaluated dynamically via a ray tracer channel simulator. Accelerated by a warm start strategy and elitist replacement, the proposed customized GA provides near-optimal results in experiments, applicable to generic environment with multiple mobile users and different signal-to-noise ratios.
\end{abstract}

Index Terms-Massive MIMO, multi-user, topology, focusing performance, radio propagation channel, ray tracer, genetic algorithm

\section{INTRODUCTION}

We are experiencing an immensely increased wireless data consumption via diverse applications and devices. Wireless networks with even lower latency, higher spectral efficiency, and higher reliability are in urgent need. The unused spectrum at higher frequencies with large bandwidth opportunities are exploited for commercial use. With higher carrier frequency, massive multiple-input multiple-output (mMIMO) is a responsive choice in order to maintain the link budget. A mMIMO system is the system with unconventionally many active antenna elements with a total number of $M$ that can serve users $K(M>>K)$ in the same time-frequency resources [1]. The ratio between $M$ and $K$ depends on the requirements of system performance, the propagation environment, and so on. For sub$6 \mathrm{GHz}$, massive multi-antenna base station (BS) with 64 or 128 antennas appeared as commercial products. For millimeterwave (mmWave) frequencies, systems equipping more than 128 antennas are foreseen. In perspective of functionality, a mMIMO system can simultaneously transmit multiple independent streams where each experiences unique and independent propagation. Therefore the spectral efficiency increases as a spatial multiplexing gain. It can also transmit coherent signals to antennas so that the signals add coherently towards the target user then the signal-to-noise ratio (SNR) increases as a beamforming gain. mMIMO is hardly a point-to-point solution and is for multi-user (MU) exploiting multiplexing with beamforming.
The spatial resolution of a mMIMO system is not only determined by the number of the BS antennas, but also the aperture size and BS-user topology [2]. Typical topology choices include the co-located, the distributed, and the cellfree [3]. For the co-located topology, the BS array is configured with closely spaced antennas with specific geometry, and there is low requirement for the backhaul network. For the split topology, the BS array is split and distributed in separated locations, and the cooperative backhaul network is necessary. For the cell-free (a.k.a. "radio stripes"), each user is essentially surrounded by BS antennas, which differs from the current commercial network deployment that each BS is surrounded by users; it also requires cooperative backhaul network. Here we are interested in the cell-free topology for MU-mMIMO.

Given certain mMIMO topology, the intrinsic challenge posing between BS and $\mathrm{MU}$ is the dispersive and directive radio propagation channel. The knowledge of the mMIMO radio channel is crucial in order to evaluate the focusing performances of BS over the spread of UE devices, with tuning of precoding techniques including the maximum ratio transmission (MRT), the regularized zero forcing (ZF) and the minimum mean squared error (MMSE). Nevertheless, the impacts of the mMIMO topology, the MU effect including mobility, and the propagation mechanism on the system focusing performance, should be understood as a whole and furthermore should be able to feedback to us the optimal deployment. The mMIMO topology shall be optimized in a smart way given the generic MU mobility and propagation channel condition; hence the goal of this paper. We propose to use the genetic algorithm (GA) combined with a ray tracer (RT) channel simulator to generate the optimal mMIMO topology serving for MU in mobility.

Belonging to the broader class of evolutionary algorithms (EA) inspired by the biological evolution, GA encodes candidate solutions using chromosomes and provides a fitness function determining their qualities [4]. Crossover and mutation are performed on chromosomes while selection is effectuated by their fitness values. GA has been applied in mMIMO technologies particularly for antenna design [5], antenna selection [6], resource allocation [7], channel estimation [8] and multiobjective optimization tasks [9]. Relevant to our work, GA has been employed in [10] to find the optimal antenna placement in a user equipment aiming for the best capacity. GA is also used 
TABLE I: Strengths and Challenges of GA and its potential on mMIMO BS antenna deployment

\begin{tabular}{|c|c|c|}
\hline & GA itself & Applying GA to mMIMO BS antenna deployment \\
\hline Strengths & $\begin{array}{l}\text { - No need of large training data compared to machine learning methods } \\
\text { - Can find better solutions beyond the initialization candidates } \\
\text { - Relatively simple to implement }\end{array}$ & $\begin{array}{l}\text { - A brand new attempt to solve the problem } \\
\text { - A plain encoding and genetic operations are proposed } \\
\text { - Applicable to generic environments }\end{array}$ \\
\hline Challenges & $\begin{array}{l}\text { - Finding a feasible encoding is sometimes difficult } \\
\text { - There exist objectives that can not be converted to fitness functions } \\
\text { - There are concerns that the algorithm not work on some problems } \\
\text { - The algorithm could trap in local optima }\end{array}$ & $\begin{array}{l}\text { - Better deployments may exist beyond the GA output } \\
\text { - How to improve the GA is worth investigation }\end{array}$ \\
\hline
\end{tabular}

in [11] for a comparison with a gravitational search algorithm (GSA) to achieve the selection of optimal transmit antennas maximizing both capacity and energy efficiency, without giving implementation details.

In this paper, our investigated problem is beyond the scope of the literature and aims at a general applicability of GA for mMIMO topology optimization given $\mathrm{MU}$ in mobility and propagation scenario with generic line-of-sight (LOS) and specular reflections (SR). Our initial focus is to optimize the BS antenna locations in a cell-free topology to achieve the optimized sum-rate capacity. To the best of our knowledge, this paper is an early effort to tackle the optimal mMIMO topology using GA combined with ray tracer. Strengths and challenges of GA and its potential in optimizing mMIMO BS antenna deployment are summarized in Table I to support our motivation statement.

\section{System Model And Focusing Performance}

\section{A. Cell-free Topology in Confined Environment}

The cell-free topology aims at providing an almostuniformly good service quality for users everywhere [3]. Ideally, there is no division of cells, but just the spread out of access points, or in other words, a massive number of distributed BS antennas. In this topology, each user is served by all the antennas in its area of influence, so that the user-centric clustering is formed to reinforce channel hardening [12], [13]. The cell-free topology requires a lot of backhaul signaling; the authors in this paper regard this cell-free topology positive and feasible to implement in large confined environment, e.g., warehouse, parking lot.

\section{B. Ray Tracer}

The 3-D RT tool used in this paper is based on [14], and can be used to capture propagation mechanisms including LOS, SR with different order, penetration, diffraction, and diffuse scattering. This RT tool has been utilized in the literature and its effectiveness has been validated in [15], [16], [17]. The input information are the geometrical and electromagnetic descriptions of the environment, including walls, ceiling, floor and furniture if any, as well as the antenna radiation properties.

\section{C. mMIMO System Model}

Considering channel reciprocity and only the downlink (DL) channel of MU-mMIMO, the BS is equipped with $M$ scattered antennas serving simultaneously $K$ single-antenna users distributed randomly in the scenario. The received DL signal for user $k$ denoted by $y_{k}$ is given by [18]:

$$
y_{k}=\sqrt{p_{k}} \boldsymbol{h}_{k} \boldsymbol{x}+n_{k}
$$

where $\boldsymbol{h}_{k} \in \mathbb{C}^{1 \times M}$ is the channel vector perceived by user $k$ and belongs to the $k$-th row of channel matrix $\boldsymbol{H} \in \mathbb{C}^{K \times M}$, $p_{k}$ is the power allocated to user $k, n_{k} \sim C N\left(0, \sigma^{2}\right)$ is the complex Gaussian noise attributed to user $k, \sigma$ is the standard deviation of the noise power, and $\boldsymbol{x} \in \mathbb{C}^{M \times 1}$ is the transmitted signal with precoders:

$$
\boldsymbol{x}=\boldsymbol{W} s .
$$

In (2), $s \in \mathbb{C}^{K \times 1}$ is pre-coded data symbol vector, $\boldsymbol{W} \in$ $\mathbb{C}^{M \times K}$ is the precoding matrix formed by the beamforming vectors for each user:

$$
\begin{aligned}
& \boldsymbol{W}=\left\{\begin{array}{cc}
\boldsymbol{H}^{H} & \text { MRT } \\
\boldsymbol{H}^{H}\left(\boldsymbol{H}^{H}\right)^{-1} & \text { ZF } \\
\boldsymbol{H}^{H}\left(\boldsymbol{H} \boldsymbol{H}^{H}+\frac{K}{\rho} \boldsymbol{I}_{k}\right)^{-1} & \text { MMSE }
\end{array},\right. \\
& \boldsymbol{W}=\frac{\boldsymbol{W}}{\|\boldsymbol{W}\|_{\mathrm{F}}}
\end{aligned}
$$

where $\{\cdot\}^{H}$ indicates the conjugate transpose, $\boldsymbol{I}_{k}$ is the $K \times K$ identity matrix, and $\rho$ is the SNR. Assuming $\boldsymbol{x}$ and $\boldsymbol{n}$ have unit power in (1), the allocated power $p$ indicates the SNR and equals to $\rho$. The use of (3) the classical linear precoder assumes that BS has the perfect CSI. The use of linear precoders is beneficial in terms of reducing the number of RF chains hence subsequently results in a decrease in hardware and software complexity. The performances of linear precoders combined with heuristic power allocation schemes are typical indicators on the effectiveness of the mMIMO strategy; such strategy is plausible for the reduced complexity compared to, e.g., dirty paper coding. In (4), the precoding matrix $\boldsymbol{W}$ is powernormalized. Combining (1), (2), (3) leads to:

$$
\begin{gathered}
y_{k}=\boldsymbol{h}_{k} \boldsymbol{w}_{k} s_{k}^{\prime}+\sum_{i=1, i \neq k}^{K} \boldsymbol{h}_{k} \boldsymbol{w}_{i} s_{i}^{\prime}+n_{k}, \\
\boldsymbol{s}^{\prime}=\sqrt{\boldsymbol{P}} \boldsymbol{s} .
\end{gathered}
$$




\section{Evaluation Metrics for $M U$}

It is crucial to evaluate the mMIMO system performance and here we adopt the sum-rate capacity as the metrics indicating the focusing performance. First, the signal power to interference ratio (SINR) is defined as:

$$
S I N R_{k}=\frac{p_{k}\left|\boldsymbol{h}_{k} \boldsymbol{w}_{k}\right|^{2}}{\sum_{i=1, i \neq k}^{K} p_{i}\left|\boldsymbol{h}_{k} \boldsymbol{w}_{i}\right|^{2}+\sigma^{2}},
$$

where

$$
p_{k}=\left\{\begin{array}{cc}
\rho M & \text { Equal power allocation } \\
p_{k}^{*} & \text { Optimized power after waterfilling. }
\end{array}\right.
$$

Then the sum-rate capacity serving as an upper bound on the achievable rates and spectral efficiency is given by:

$$
\begin{gathered}
C=\sum_{k=1}^{K} C_{k}, \\
C_{k}=\log _{2}\left(1+S I N R_{k}\right)[b i t / s / H z] .
\end{gathered}
$$

Note that the achievable rate depends on the topology as well as the propagation channel.

\section{Genetic Algorithm For Optimization}

We propose a customized GA (CGA) as described in Algorithm 1 to specially solve the aforementioned mMIMO deployment optimization problem. This section provides a general description of the proposed scheme and Section IV dicusses the numerical investigations. In the proposed scheme using GA combined with RT, a binary matrix encoding is adapted to the candidate solutions enabling further employment of genetic operations.

In the initialization stage, the first population is generated by grouping individuals where each individual encodes the randomly chosen $M$ antenna locations. The individuals are sorted by their fitness (performance), which is the sum-rate capacity presented in Section II. The algorithm keeps running until the stop condition [19] (specific execution time, iteration number or fitness value) is reached. A warm start strategy named truncation update [20] is introduced at the beginning of each iteration, e.g., the bottom $50 \%$ individuals in the fitness ranking are replaced by the individual with the best fitness. Afterwards, roulette wheel selection [21] is performed on the current population choosing two individuals named parents. Single point crossover [22] is conducted on parents generating two children, where bit flip mutation [23] is applied. An intuitive example for the above mentioned genetic operations is given as follows: suppose two parents are selected in one iteration, denoted by $p_{1}=[110011]$ and $p_{2}=$ [101001]. The crossover point is after column 3 , therefore two children $c_{1}=[110 \mid 001]$ and $c_{2}=[101 \mid 011]$ are generated by changing the second half of two parents. The mutation point is column 2 , therefore $c_{1}=\left[1 \underline{00001]}\right.$ and $c_{2}=[1 \underline{11011]}$ are updated by bit filp on column 2. Finally, elitist replacement [24] is implemented ensuring the two best in parents and children would replace the selected parents. The updated population is further sorted for the next iteration by fitness.

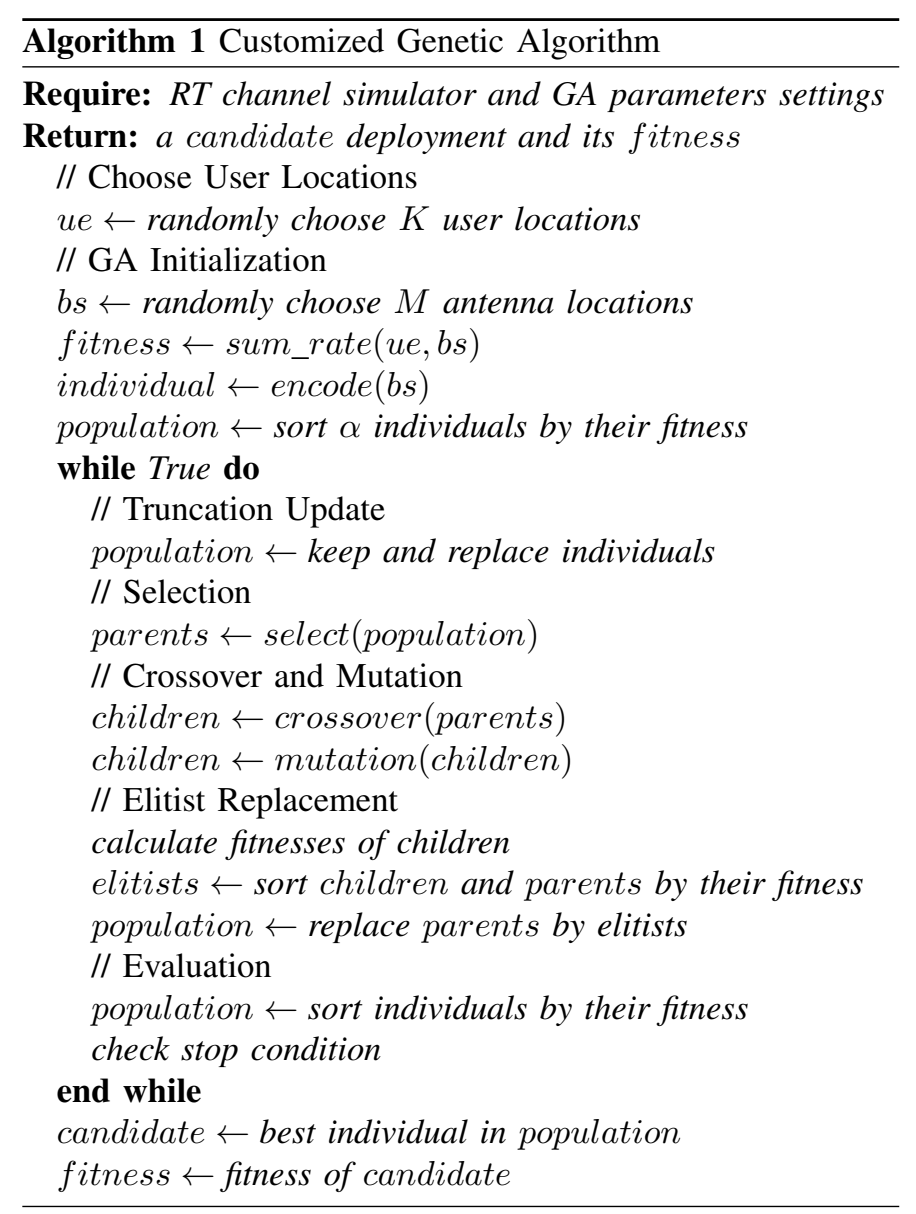

Considering the fitness function has the high time complexity of $O(K * M)$ (calculating the sum_rate), in the implementation of CGA, fitnesses are calculated once and stored in an additional sorted array, whose index is used for sorting individuals. For a specific MU group $u e$, with the population size $\alpha$ and a maximal iteration number $N$, the CGA explores $N *(\alpha+2)$ candidate deployments of BS antennas using the RT simulator. The candidate with the best performance is returned as the CGA output.

\section{NUMERICAL INVESTIGATIONS}

We apply the following setups for the numerical experiments of the proposed CGA scheme in Section III. The confined environment is a cubic (representing a room) with length, width, and height as 5 meters. The walls are reflective and lossy bricks. There is no scattering from dryground and the ground is not penetrable. The dielectric and conductivity properties of walls, ceiling and dryground follow the ITU recommendations [25]. The target frequency is $5.9 \mathrm{GHz}$ with $200 \mathrm{MHz}$ bandwidth. The BS antennas are half-wavelength vertical dipoles and each UE is one single dipole antenna as well. The LOS and the 1st order SRs are captured in RT for the radio channel between $\mathrm{BS}$ antennas and UE antnenas. In this confined environment, the $K$ users are randomly situated in the horizontal plane with height of 1.6 meters; the $M \mathrm{BS}$ antennas are assumed to be equally distributed on four walls 
surrounding users whearas the locations of the BS antennas are randomly generated on the vertical walls. To evaluate the sum-rate capacity, we assume a certain level of SNR and SNR is variable. We use MMSE and equal power allocation. Note that the priority of this paper is not to find the best power allocation and precoding scheme, but rather to investigate the applicability of CGA on optimizing cell-free MU-mMIMO. The GA population size $\alpha$ is set to 4 , and the maximal iteration number $N=10$ is chosen as the stop condition.

The CGA encoding procedure is performed as follows. BS antenna positions on each wall have a fixed value in one dimension of the 3D Cartesian coordinates (Fig. 3). The location of an antenna is represented using a binary matrix of 2 rows and 6 columns, with the locating precision of $5 / 64$ meters. Each row is a binary representation of the decimal value indicating its relative position in one dimension.

In the first experiment the CGA is executed on the scenario with $K=5$ users at fixed locations (denoted as $u e 1$ ), $M=20$ BS antennas, and SNR of $30 \mathrm{~dB}$. The search trend of CGA is shown in Fig. 1, where the mean and the best fitness (performance in sum-rate capacity) values of each iteration are captured. The figure indicates the convergence of the algorithm, with the increasing mean fitness over iterations. Compared to the traditional GA (TGA) [26] without the warm start strategy of truncation update, the CGA has a faster convergence speed and provides a better result after 10 iterations, since the quality of initial individuals in each iteration are improved. The box plots in Fig. 2 further demonstrate the convergence of fitness in a detailed view over iterations. With the elitist replacement strategy, final individuals after evolution (truncation update, crossover, mutation, elitist replacement) outperform the initial ones in all iterations. Upon iteration termination, a near-optimal deployment of BS antenna locations denoted as $d p 1$ is found with performance converged at 4.56 $\mathrm{bit} / \mathrm{s} / \mathrm{Hz}$, denoted as $f 1$. In this experiment the CGA explores $10 *(4+2)=60$ candidates and returns the best one. We further evaluate $d p 1$ with $p$ groups of 5 randomly generated users, simulating the scenario of generic mobile users. For $p=5$, calculated by the RT simulator without the CGA execution, performances $\left(\left[\right.\right.$ sum_rate $\left.\left.\left(u e 1_{i}, d p 1\right)\right], i \in[1 . . p]\right)$ are $[4.52,4.55,4.54,4.54,4.54] \mathrm{bit} / \mathrm{s} / \mathrm{Hz}$, relatively good compared to $f 1$.

In the second experiment the CGA is applied to $q$ groups of 5 random users with $20 \mathrm{BS}$ antennas. Therefore, the algorithm runs $q$ epochs in such experiment rather than one in the first experiment. Each epoch on our working desktop (Intel i7 7700, 16G RAM with Matlab R2017b) the CGA requires about 3 hours. For $q=2$, none of the result ((ue2,dp2), (ue3,dp3) with $[3.04,3.77] \mathrm{bit} / \mathrm{s} / \mathrm{Hz})$ from those epochs has a converged performance outperforming $d p 1$. This phenomenon indicates that for the generic environment " $K$ mobile users with $M$ antennas", we could apply the CGA within one epoch on $K$ randomly situated users to achieve an acceptable and near-optimal $M$ antenna deployment. All users and antenna locations are visualized in Fig. 3. Compared with an arbitrary deployment ( $d p 4$ with $2.83 \mathrm{bit} / \mathrm{s} / \mathrm{Hz}$ ), our method could provide

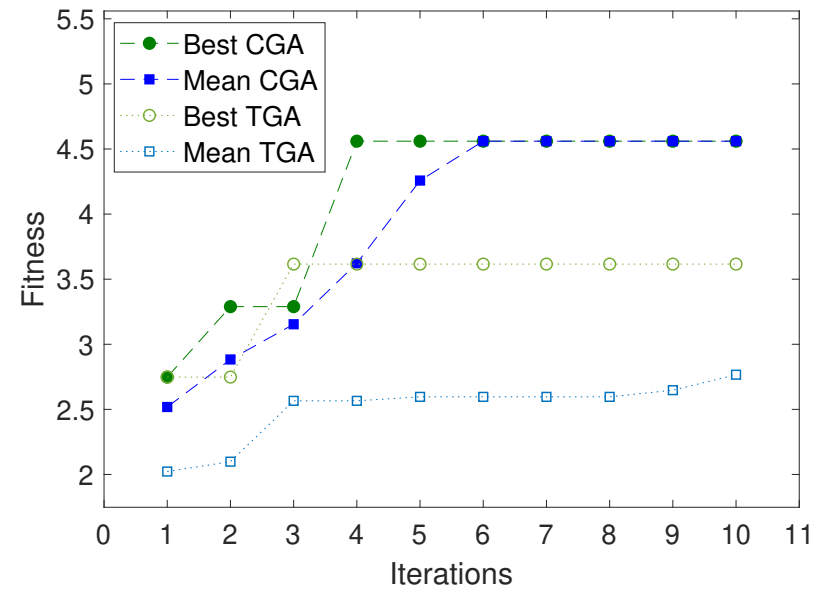

Fig. 1: Search trend for CGA and TGA in experiment 1

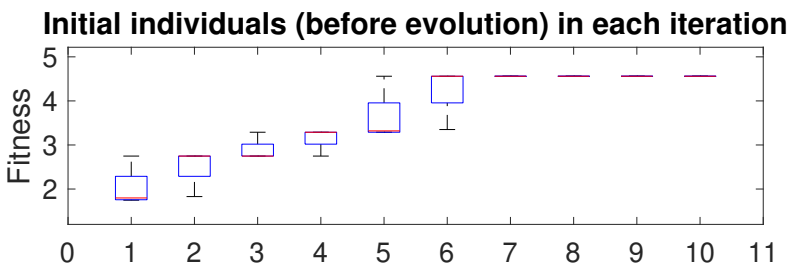

Final individuals (after evolution) in each iteration

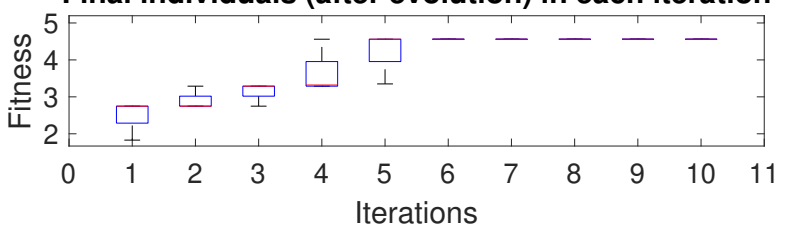

Fig. 2: Box plots for each iteration of CGA in experiment 1

solutions with better performances ( $7.4 \%$ with $d p 2,33.2 \%$ with $d p 3$, and $61.1 \%$ with $d p 1$ ). Conventional approach to mMIMO antenna deployment are based on empirical analyses, with our method more competitive candidates are provided. Additional experiments of CGA applications on scenarios with different SNRs are also performed. The same antenna deployment is produced for different SNR scenarios when other settings keep the same.

Tuning GA parameters by applying larger population size $\alpha$ and maximal iteration number $N$ could improve the performance of CGA, resulting in a faster convergence speed or a better solution. Adjusting the truncation, crossover, and mutation rate also has notable influence on the search progress [27]. However, according to the no free lunch theorem [28], better performance also requires higher execution cost, e.g. time consumption in this case. The proposed CGA is designed with truncation update and elitist replacement, accelerating the convergence speed without significantly increasing the running time. 


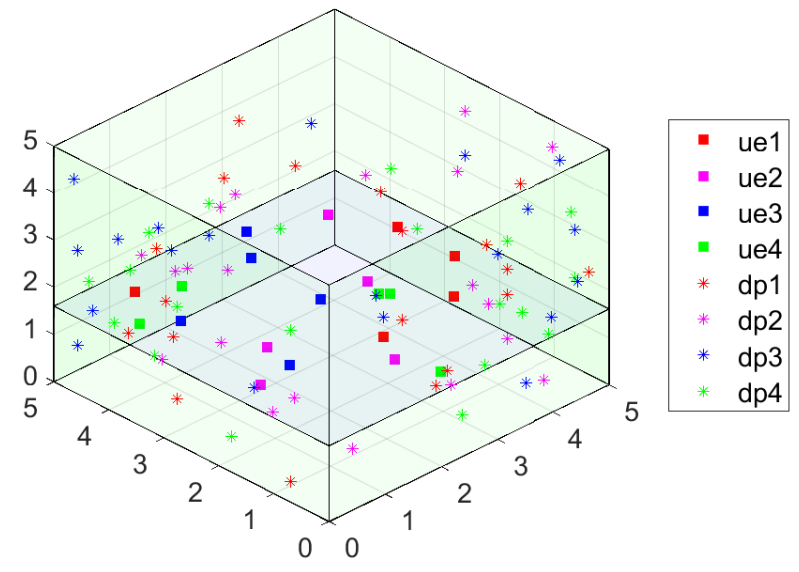

Fig. 3: Positions of users and antennas

\section{CONCLUSION}

This paper presents a customized genetic algorithm and its application to the problem of massive MIMO cell-free topology optimization. The genetic algorithm is combined with a ray tracer simulator as a dynamic measure of performances for candidate deployments for base station antenna placement. Elitists outperforming the initial individuals over iterations of the CGA guarantee the effective search of candidate solutions. Numerical investigations indicate the general applicability of the algorithm in different settings in a confined environment where radio propagation is dominant by line-of-sight and specular reflection. Further research and development include how to make the algorithm converge fast in dynamic environment scenarios, and how this convergent near-optimal approaches the reality.

\section{REFERENCES}

[1] E. Bjornson, E.G. Larsson, T.L. Marzetta, "Massive MIMO: ten myths and one critical question," IEEE Communication Magazine, vol. 54, no. 2, pp. 114-123, Feb. 2016.

[2] E. Bjornson, L. Sanguinetti, H. Wymeersch, J. Hoydis, T.L. Marzetta, "Massive MIMO is a reality - what is next? Five promising research directions for antenna arrays," arXiv:1902.07678v1 [eess.SP], Feb. 2019.

[3] H.Q. Ngo, A. Ashikhmin, H. Yang, E.G. Larsson, T.L. Marzetta, "Cellfree massive MIMO versus small cells," IEEE Transactions on Wireless Communication, vol. 16, no. 3, pp. 1834-1850, Jan. 2017.

[4] W. Darrell, "A genetic algorithm tutorial," Statistics and computing, vol. 4 , no. 2, pp. $65-85,1994$

[5] M.O. Binelo, A.L.F. de Almeida, F.R.P. Cavalcanti, "MIMO Array Capacity Optimization Using a Genetic Algorithm," IEEE Transactions on Vehicular Technology, vol. 60, no. 6, pp. 2471-2481, July 2011.

[6] B. Makki, A. Ide, T. Svensson, T. Eriksson, M. Alouini, "A Genetic Algorithm-Based Antenna Selection Approach for Large-but-Finite MIMO Networks," IEEE Transactions on Vehicular Technology, vol. 66, no. 7, pp. 6591-6595, July 2017.

[7] N. Sharma, K.R. Anupama, "A novel genetic algorithm for adaptive resource allocation in MIMO-OFDM systems with proportional rate constraint," Wireless Personal Communications, vol. 61, no. 1, pp. 113128, Jan. 2011

[8] X. Ma, F. Yang, S. Liu, J. Song, Z. Han, "Design and Optimization on Training Sequence for mmWave Communications: A New Approach for Sparse Channel Estimation in Massive MIMO," IEEE Journal on Selected Areas in Communications, vol. 35, no. 7, pp. 1486-1497, July. 2017
[9] S.K. Goudos, P.D. Diamantoulakis, G.K. Karagiannidis, "Multi-Objective Optimization in 5G Wireless Networks With Massive MIMO," IEEE Communications Letters, vol. 22, no. 11, pp. 2346-2349, Nov. 2018.

[10] P. Uthansakul, D. Assanuk, M. Uthansakul, "The use of Genetic Algorithm for designing MIMO antenna placement," 2010 International Conference on Computer and Information Application, Tianjin, 2010, pp. 414-417.

[11] I.V. RAO, V.M. RAO, "Optimal Transmit Antenna Selection Using Improved GSA in Massive MIMO Technology," Wireless Personal Communications vol. 109, pp. 1217-1235, May 2019.

[12] A.O. Martinez, E.D. Carvalho, J.O. Nielsen, "Massive MIMO properties based on measured channels: channel hardening, user decorrelation and channel sparsity," in 2016 50th Asilomar Conference on Signals, Systems and Computers, Pacific Grove, CA, USA, Nov. 2016.

[13] Y. Miao, S. Pollin and A.A. Glazunov, "Simulation-based Investigation on Massive Multi-Antenna System as to Spatial Channel Hardening for Mobile Single User in a Controlled Multipath Environment," 2020 14th European Conference on Antennas and Propagation (EuCAP), Copenhagen, Denmark, 2020.

[14] F. Mani, "Improved ray-tracing for advanced radio propagation channel modeling," Ph.D. dissertation, Univ. Catholique de Louvain, OttigniesLouvain-la-Neuve, Belgium, Jun. 2012

[15] F. Mani, F. Quitin, and C. Oestges, "Directional spreads of dense multipath components in indoor environments: Experimental validation of a ray-tracing approach," IEEE Trans. Antennas Propag., vol. 60, no. 7, pp. 3389-3396, Jul. 2012.

[16] Y. Miao, T. Pedersen, M. Gan, E. Vinogradov and C. Oestges, "Reverberant Room-to-Room Radio Channel Prediction by Using Rays and Graphs," IEEE Transactions on Antennas and Propagation, vol. 67, no. 1, pp. 484-494, Jan. 2019

[17] Y. Miao, Q. Gueuning, M. Gan and C. Oestges, "Adding diffuse scattering correlation to effective roughness models in ray tracing," 2017 11th European Conference on Antennas and Propagation (EUCAP), Paris, France, Mar. 2017.

[18] T. Marzetta, E. Larsson, H. Yang, H. Ngo, Fundamentals of Massive MIMO. Cambridge: Cambridge University Press, 2016.

[19] K. Shen, J. David, T.D. Pessemier, L. Martens, W. Joseph, "An efficient genetic method for multi-objective continuous production scheduling in Industrial Internet of Things," 2019 24th IEEE International Conference on Emerging Technologies and Factory Automation (ETFA), Zaragoza, Spain, 2019, pp. 1119-1126.

[20] I. Harvey, "The microbial genetic algorithm", European Conference on Artificial Life, Berlin, Heidelberg, 2009, pp.126-133.

[21] A. Lipowski, D. Lipowska, "Roulette-wheel selection via stochastic acceptance," Physica A: Statistical Mechanics and its Applications, vol. 391, no. 6, pp. 2193-2196, Mar. 2012.

[22] O. Hasançebi, F. Erbatur, "Evaluation of crossover techniques in genetic algorithm based optimum structural design," Computers \& Structures vol. 78 , no. $1-3$, pp. 435-448, Nov. 2000.

[23] F. Ye, C. Doerr, T. Bäck, "Interpolating Local and Global Search by Controlling the Variance of Standard Bit Mutation," 2019 IEEE Congress on Evolutionary Computation (CEC), Wellington, New Zealand, 2019, pp. 2292-2299.

[24] C.F. Lima, M. Pelikan, D.E. Goldberg, F.G. Lobo, K. Sastry, M. Hauschild, "Influence of selection and replacement strategies on linkage learning in BOA," 2007 IEEE Congress on Evolutionary Computation, Singapore, 2007, pp. 1083-1090.

[25] "Effects of building materials and structures on radiowave propagation above about $100 \mathrm{MHz}$ International Telecommunication Union - Radiocommunication Sector (ITU-R)", 2040-1 P-Series Radiowave Propagation, Jul. 2015.

[26] K. Shen, T.D. Pessemier, X. Gong, L. Martens, W. Joseph, "Genetic Optimization of Energy- and Failure-Aware Continuous Production Scheduling in Pasta Manufacturing," Sensors, vol. 19, no. 297, Jan. 2019.

[27] M. Srinivas, L. M. Patnaik, "Adaptive probabilities of crossover and mutation in genetic algorithms," IEEE Transactions on Systems, Man, and Cybernetics, vol. 24, no. 4, pp. 656-667, Apr. 1994.

[28] D.H. Wolpert, W.G. Macready, "No free lunch theorems for optimization," IEEE Transactions on Evolutionary Computation, vol. 1, no. 1, pp. 67-82, April. 1997. 